\title{
Study of variant superficial palmar arch entirely formed by the ulnar artery
}

\author{
Sonal Govindwar ${ }^{1}$, Swapna Chavan ${ }^{2 *}$ \\ ${ }^{1}$ Assistant Professor, Nanded Rural Dental College \& Research Center, Nanded, Maharashtra, INDIA. \\ ${ }^{2}$ Assistant Professor, Department of Anatomy, HBT Medical College, and Coopar Hospital, Vile Parle, West Mumbai, Maharashtra, INDIA. \\ Email: sonal.govindwar@gmail.com, swapna.chavan2012@gmail.com
}

Abstract Superficial Palmar Arch is an arterial arcade and a dominant vascular structure in the palm. It is defined as the anastomosis between the superficial branch of the ulnar artery and superficial palmar branch of the radial artery. In ulnar dominant complete superficial palmar arch, the ulnar artery does not anastomose with radial artery and it terminates by supplying thumb and index finger. In the present study, this type of variation was found in $30.43 \%$ of the hands. This is in contrast to the classical superficial palmar arch normally described where the arch is completed on the radial side by superficial palmar branch of radial artery. In accordance with the present study, a feature that is present in $50 \%$ of the specimens cannot be called as a rare. In these cases, without an efficient collateral circulation, ulnar artery occlusion may cause claudication and gangrene in the digits and has clinical significance.

Key Words: Superficial palmar arch, ulnar dominant complete superficial palmar arch, collateral circulation, anastomosis.

*Address for Correspondence:

Dr. Swapna Chavan, Assistant Professor, Department of Anatomy, HBT Medical College, and Coopar Hospital, Vile Parle, West Mumbai, Maharashtra, INDIA.

Email: swapna.chavan2012@gmail.com

Received Date: 08/04/2017 Revised Date: 10/05/2017 Accepted Date: 18/06/2017

DOI: https://doi.org/10.26611/1001411

\begin{tabular}{|l|l|}
\hline \multicolumn{2}{|c|}{ Access this article online } \\
\hline Quick Response Code: & Website: \\
\hline & www.medpulse.in \\
\hline
\end{tabular}

INTRODUCTION

Superficial Palmar Arch is an arterial arcade and a dominant vascular structure in the palm. It is defined as the anastomosis between the superficial branch of the ulnar artery and superficial palmar branch of the radial artery. In ulnar dominant complete superficial palmar arch, the arch is entirely formed by ulnar artery and supplies thumb and index finger without communicating with radial artery. In these cases, potential hazard could exist to the digits including thumb and index finger in the event of traumatic injury to the ulnar artery. ${ }^{1}$ Due to its superficial nature, the arterial arch is too constantly exposed to mechanical injuries ${ }^{2}$. The ulnar supplied superficial palmar arch, when interrupted, is most likely to cause digital symptoms ${ }^{3}$. Jaschtschinski SN $(1896)^{4}$ in his study of 200 hands, found the arch formed entirely by ulnar artery which supplies thumb and index finger in $38 \%$ of the specimens. Coleman SS, Anson BJ (1961) found the incidence of complete arch formed entirely by ulnar artery in 37\%. MAJ Mozersky DJ et al (1973) ${ }^{6}$ noted the ulnar dominant arch in $88 \%$ of the hands they studied by ultrasonic evaluation in 70 young volunteers. Earley MJ (1986) ${ }^{7}$ noted the incidence of complete arch formed entirely by ulnar artery in $20 \%$ of the hands. Gellman $\mathrm{H}$ et al $(2001)^{8}$ found the incidence of complete arch formed entirely by ulnar artery in $31.1 \%$ of the hands. The incidence of complete arch formed entirely by ulnar artery is seen to vary from 20 to $88 \%$, though this is not the classical pattern normally described. The present study was to evaluate the $\%$ of cases in which ulnar artery forms the arch entirely and supplies the thumb and index finger. In these cases without an efficient collateral circulation, ulnar artery occlusion may cause claudication and gangrene in the digits and has clinical significance. Recent improvements in microsurgical techniques have increased the necessity of better understanding of the vascular pattern of the hand. 


\section{MATERIALS AND METHODS}

A total of 46 embalmed adult human cadaver hands were dissected. A horizontal incision was made at the wrist joint. A vertical incision was made from the middle of this incision upto the 3rd metacarpophalangeal joint. A horizontal incision was made along the root of the fingers. The skin of the palm was reflected. The palmar aponeurosis was removed to reveal the superficial palmar arch and its branches. The arch and its branches were clearly demonstrated by the dissection of the surrounding adipose tissue and the removal of the digital branches of median and ulnar nerves from the palm. The arches were painted in red using artist oil colour. The arches formed entirely by the ulnar artery without anastomosing with radial artery, and supplying the thumb and index finger were noted.

\section{RESULTS}

Complete superficial palmar arch formed entirely by ulnar artery and supplying the thumb and index finger was found in 14 out of 46 hands with an incidence of $30.43 \%$. (Fig.1and 2). This is contrast to the classical superficial palmar arch normally described, where the arch is completed on the radial side by superficial palmar branch of radial artery. A feature that is present in $30.46 \%$ of the hands may not be called as a variation.

\begin{tabular}{cc} 
Table 1: Observation of percentage of ulnar type of superficial palmar arch in respective studies \\
\hline authors & $\%$ of specimens with ulnar dominant complete arch \\
\hline Jasctschinski SN $1986^{4}$ & $38 \%$ \\
Coleman SS and Anson BJ $1961^{5}$ & $37 \%$ \\
MAJ Mozersky DJ et al ${ }^{6}$ & $88 \%$ \\
Earley MJ ${ }^{7} 1986$ & $20 \%$ \\
Gellman H et al ${ }^{8} 2001$ & $31 \%$ \\
Present study & $30.43 \%$ \\
\hline
\end{tabular}



Figure 1

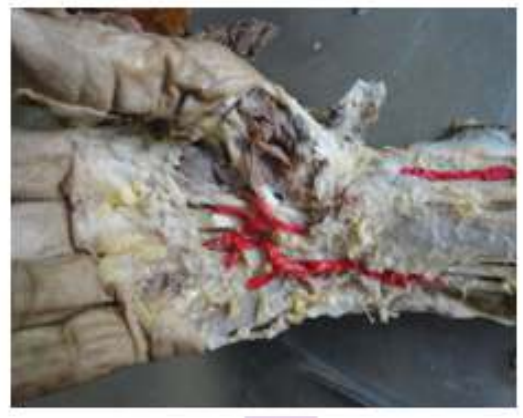

Figure 2



Figure 3

Legend

Figure 1: Incomplete superficial palmar arch; Figure 2: Incomplete superficial palmar arch; Figure 3: Incomplete superficial palmar arch entirely formed by the superficial branch of the ulnar artery.

\section{DISCUSSION}

Coleman SS and Anson BJ (1961) $)^{5}$ classified the arches into complete arches, when the contributing arteries to the superficial palmar arch anastomosed with each other or the ulnar artery itself forms the arch and supplies thumb and index finger. They grouped the complete arch formed by ulnar artery as Complete Type B and found it in $37 \%$ of the cases. The present study follows their grouping of complete arches. Tandler $\mathrm{J}(1897)^{9}$ in a study of 130 specimens found that the final termination of superficial branch of ulnar artery in the palm is usually an artery which supplies both radial side of index finger and ulnar side of the thumb. He called this as first common volar digital artery. Coleman SS and Anson $\mathrm{BJ}^{5}$ supported their study. The present study is in accordance with their observations. Little JM et al $(1973)^{10}$ noted that the circulatory dynamics in the hand follow a very variable pattern, the ulnar artery being the dominant source of supply in most hands. They concluded that collateral circulation in the hand would generally maintain viability of fingers when either radial or ulnar artery was occluded at the wrist. However, they found that in $9 \%$ of the hands, there was drastic disturbance of circulation on occlusion of ulnar artery. They suggested that the variation in collateral adequacy in the hand may explain ischaemic phenomena seen in some patients with the hammer hand syndrome. Hypothenar hammer syndrome occurs in persons who use hand as a hammer. Any finger could become ischaemic following occlusion of ulnar artery or superficial volar arch depending upon the pattern of branching present in the superficial arch and the distribution of digital arteries Conn J et al $(1970)^{11}$. 
Loukas M et al (2009) ${ }^{1}$ reported a case of complete superficial palmar arch which had no contribution from radial artery and terminated by giving rise to a common trunk for the princeps pollicis and radialis indicis arteries. They stated that as the arterial supply of thumb in this case is solely provided by superficial palmar arch, a potential hazard could exist in the event of traumatic injury to the ulnar artery. Mookambica RV et al (2010) ${ }^{12}$ reported a case where the superficial palmar arch was formed exclusively by superficial branch of ulnar artery, without contribution by any other vessel. Ulnar artery continued as the first common digital artery to the interdigital cleft between index finger and thumb and this digital artery was dividing into APP and ARI. They called this type of arch as incomplete SPA based on Gellman classification of palmar arches. They stated that the nomenclature of arteries originating from SPA and supplying thumb and index finger have to be discussed because of their surgical importance. In hand surgeries like vascular graft applications, arterial repairs, free/pedicle flaps clinicians should be aware of these variations, because in most of the traumatic events and the surgical procedures of the hand, SPA plays an important role. In case of ulnar skin flaps, damage to the ulnar artery may present a risk. Interference with an efficient blood supply may result in inefficient utility of the movements of fingers and hand. Superficial Palmar Arch is the main vascular structure of palm. Hence knowledge about the variation in its pattern is important for surgeons dealing with reconstructive hand surgeries and restoration of functional anatomy of hand. To conclude, it is important to continually report significant anatomical variations so that clinicians are aware of these anomalies and thus avoid the potential.

\section{REFERENCES}

1. Loukas M., Tubbs S., Louis Jr. R.G., Apaydin N. Princeps Policies artery arising from the superficial palmar arch. Singapore Med J 2009; 50 (11):e391-392.

2. Moore L.K., Dalley AF. Clinically oriented anatomy. 4th ed. William and Wilkins, Philadelphia, Lippincott 1999 , pp773-774.

3. Silcott G.R., Polich V.L. Palmar arch arterial reconstruction for the salvage of ischemic fingers. Am J Surg 1981; 142: 219-225.

4. Jaschtschinski S.N. Morphologie and topographie des arcus volaris sublimis and profundus. Anat Hefte 1897; 7:163-188.

5. Coleman S.S., Anson B.J. Arterial patterns in the hand based upon a study of 650 specimens. Surg Gynecol Obstet 1961; 113: 409-424.

6. Maj Mozersky D.J., Lt. Col. Buckley C.J., Col. Hagood, Jr., Col. Capps W.F., Jr., Col. Danne Miller F.J. Jr. Ultrasonic evaluation of palmar circulation. A useful adjunct to radial artery cannulation. The Am J of Surgery 1973; 126: 810-812.

7. Earley M.J. The arterial supply of the thumb, first web and index finger and its surgical application. J Hand Surg 1986; II B(2): 163-174.

8. Gellman H., Botte M.J., Shankwiler J., Gelberman R.H. Arterial patterns of the deep and superficial palmar arches. Clin Orthop and Related Research 2001; 383: 4146.

9. Tandler J. Zur Anatomie der arterien der hand. Anat Hefte 1897; 7: 263-282.

10. Little J.M., Zylstra P.L., West J., My J. Circulatory patterns in the normal hand. Brit J Surg 1973; 60: 652655.

11. Conn J., Bergan J.J., Bell J.L. Hypothenar hammer syndrome post traumatic digital ischemia. Surgery 1970; 68(6): 1122-1128Little J.M., Zylstra P.L., West J., May J. Circulatory patterns in the normal hand. Brit J Surg 1973; 60: 652-655.

12. Mookambica R.V., Nair V., Nair N., Somayaji S.N., Pamidi N., Vollala V.R. Incomplete superficial palmar arch. International Journal of Anatomical Variations 2010; 3: 65-66.

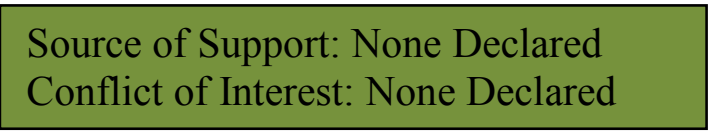

